\title{
Meeting the Challenge of Virtual Diabetes Care: A Consensus Viewpoint on the Positioning and Value of Oral Semaglutide in Routine Clinical Practice
}

\author{
Marc Evans · Angharad R. Morgan · Stephen C. Bain • \\ Sarah Davies · Debbie Hicks · Pam Brown · Zaheer Yousef · \\ Umesh Dashora · Adie Viljoen · Hannah Beba · W. David Strain
}

Received: December 1, 2021 / Accepted: December 24, 2021 / Published online: January 19, 2022

(C) The Author(s) 2022

\begin{abstract}
While glucagon-like peptide-1 receptor agonists (GLP-1 RAs), such as semaglutide, are among the most effective drugs for treating people with type 2 diabetes (T2D), they are clinically underutilised. Until recently, the only route for semaglutide administration was via subcutaneous injection. However, an oral formulation of semaglutide was recently licensed, with the potential to address therapy inertia and increase
\end{abstract}

M. Evans ( $\square)$

Diabetes Resource Centre, University Hospital Llandough, Penlan Road, Llandough, Cardiff CF64 2XX, UK

e-mail: marclyndon1@hotmail.com

A. R. Morgan

Health Economics and Outcomes Research Ltd., Cardiff, UK

S. C. Bain

Diabetes Research Unit, Swansea University Medical School, Swansea, UK

\section{S. Davies}

Woodlands Medical Centre, Cardiff, UK

D. Hicks

Medicus Health Partners, Enfield, London, UK

P. Brown

SA1 Medical Centre, Swansea, UK patient adherence to treatment, which is essential in controlling blood glucose and reducing complications. The availability of oral semaglutide provides a new option for both clinicians and patients who are reluctant to use an injectable agent. This has been of particular importance in addressing the challenge of virtual diabetes care during the COVID-19 pandemic, circumventing the logistical problems that are often associated with subcutaneous medication administration. However, there remains limited awareness of the clinical and economic value of oral semaglutide in routine

Z. Yousef

Wales Heart Research Institute, Cardiff University, Cardiff, UK

U. Dashora

East Sussex Healthcare NHS Trust, Seaford, UK

A. Viljoen

East and North Hertfordshire NHS Trust, Stevenage, UK

H. Beba

County Durham and Darlington NHS Foundation

Trust, Durham, UK

W. D. Strain

Diabetes and Vascular Research Centre, University of Exeter Medical School, Exeter, UK

\section{W. D. Strain}

The Academic Department of Healthcare for Older Adults, Royal Devon and Exeter Hospital, Exeter, UK 
clinical practice. In this article, we present our consensus opinion on the role of oral semaglutide in routine clinical practice and discuss its value in reducing the burden of delivering diabetes care in the post-COVID-19 pandemic period of chronic disease management.

Keywords: Glucagon-like peptide-1 receptor agonists; Oral semaglutide; Type 2 diabetes

\section{Key Summary Points}

The need for subcutaneous injection of glucagon-like peptide-1 receptor agonists (GLP-1 RAs) has been a barrier to the use of these drugs for some people with type 2 diabetes (T2D) and the healthcare professionals involved in their care.

Oral semaglutide presents an additional option for the management of T2D, which, by removing the injectable barrier, will enable access to the therapeutic benefits of GLP-1RAs to many more people with T2D.

Oral semaglutide could help to address delays in treatment intensification, improve patient adherence and help individuals reach their treatment goals.

The availability of oral semaglutide has been of particular importance in addressing the challenge of virtual diabetes care during the COVID-19 pandemic, circumventing the logistical problems that are often associated with subcutaneous medication administration.

\section{INTRODUCTION}

Type 2 diabetes (T2D) is a major public health crisis, with an estimated 463 million adults currently affected worldwide and a projected increase to 578 million by 2030, and 700 million by 2045 [1]. Poorly controlled glycaemia and delays in treatment intensification increase the risk of related microvascular complications and elevate the risk of cardiovascular (CV) disease in people with T2D $[2,3]$. Only $50 \%$ of adults with T2D in the UK achieved their glycated haemoglobin (HbA1c) target in 2020, and only $36 \%$ achieved all HbA1c, cholesterol and blood pressure targets [4]. Consequently, a large proportion of the $\mathrm{T} 2 \mathrm{D}$ population are at increased risk of diabetes-related complications, despite the number of treatments currently available.

Glucagon-like peptide-1 receptor agonists (GLP-1 RAs) are among the most effective drugs for treating people with T2D, providing good glycaemic control with a low risk of hypoglycaemia in those for whom other oral anti-diabetic drugs (OADs), such as metformin, have failed to adequately control their condition $[5,6]$. However, despite a wealth of evidence in relation to the efficacy, safety and potential CV outcome benefits, GLP-1 RAs are under-utilised [7]. While perceived high acquisition cost (albeit at a cheaper cost per unit HbA1c reduction than dipeptidyl peptidase-4 [DPP-4] inhibitors) and positioning in clinical guidelines are contributory factors, reluctance to use subcutaneous injectable medications may also play a significant role, with as many as $49 \%$ of general practitioners in the UK reporting that the injectable route of administration is a barrier to prescribing a GLP-1 RA [8]. There is also the consideration of time and resources spent by healthcare personnel in educating people with T2D about initiating injectable therapies, which has become more apparent with virtual clinics replacing traditional face-to-face diabetes clinics as a consequence of the COVID-19 pandemic in order to minimise inter-individual contact within surgeries [9].

Virtual clinics for remote consultations present multiple clinical and therapeutic challenges, including ensuring an equitable access to necessary technologies, difficulties in performing key health checks (such as testing blood pressure, HbA1c or urine sampling) [10], maintenance of patient relationships and difficulties in patient education about injectable therapies [11]. These issues have likely led to significant delays in treatment 
intensification, lack of patient adherence and fewer people reaching their treatment goals.

It is well recognised that therapy adherence tends to be greater with oral as opposed to injectable therapies in people living with diabetes [12]. Oral semaglutide, the first oral GLP-1 RA, has demonstrated superior glycaemic control compared with other oral therapies and equivalent metabolic effects compared to oncedaily liraglutide (a comparator GLP-1 RA), along with the benefit of weight loss with a once-daily pill [13-15]. Oral semaglutide was approved for the treatment of T2D in adults by the European Medicines Agency (EMA) in June 2020 and consequently was made available across all 27 European Union member states and the UK. As such, patients and clinicians who may be reluctant to initiate an injectable drug for T2D may benefit from the ease of drug initiation with oral semaglutide. This is especially important during the COVID-19 pandemic as healthcare providers can prescribe the drug virtually without worrying about the logistics of starting a person with T2D on a new route of medication administration, such as attempting to teach the injection technique virtually or requiring a clinic visit for that purpose. However, there remains limited awareness of the clinical and economic value of oral semaglutide to healthcare system stakeholders involved in the management of T2D. In this article we develop a consensus opinion on the role of semaglutide in routine clinical practice and discuss the value of oral semaglutide with respect to the holistic healthcare system in terms of providing a potential solution to the burden of diabetes care in a post-COVID-19 pandemic period of chronic disease management.

This article is based on previously published studies and does not contain any new studies with human participants or animals performed by any of the authors.

\section{THE ROLE OF ORAL SEMAGLUTIDE IN THE MANAGEMENT OF T2D}

The GLP-1 RAs are effective glucose-lowering agents that are a standard feature in T2D treatment guidelines, including the National Institute for Health and Care Excellence (NICE) guidelines which recommend that GLP-1 RAs are only prescribed after oral treatment failure and if weight loss is a desirable outcome [16]. The current NICE guidelines are considered by many to be restrictive and out of alignment with contemporary practice as they fail to take into account the full benefits of GLP-1 RAs. This is a consequence of these guidelines being published prior to $\mathrm{CV}$ benefit being demonstrated with liraglutide, dulaglutide and semaglutide. However, it should be noted that these guidelines are presently being updated, with planned publication in 2022, and that this update is expected to better align with international consensus on GLP-1 RA positioning.

The Cardiology Renal and Metabolic (CaReMeUK) group recommends GLP-1 RAs as a second-line therapy following metformin treatment failure in people with T2D who have atherosclerotic cardiovascular disease (ASCVD), including cardiac, peripheral artery and cerebrovascular disease; chronic kidney disease (CKD); and insufficiently controlled HbA1c targets [17]. The European Society of Cardiology (ESC), in collaboration with the European Association for the Study of Diabetes (EASD), recommends GLP-1 RAs, including oral semaglutide, as a first-line treatment in persons with T2D and CV disease at high risk of $\mathrm{CV}$ events-they are otherwise recommended after failure of metformin monotherapy to adequately control blood glucose levels [18]. The American Diabetes Association (ADA), also in collaboration with EASD, recommends GLP-1 RAs in people with T2D with a compelling need to minimise weight gain/promote weight loss or to minimise hypoglycaemia or with established $\mathrm{CV}$ disease, as a second-line therapy following metformin monotherapy [19].

Semaglutide improves hyperglycaemia by stimulating beta-cell insulin secretion and reducing alpha-cell production of glucagon secretion in both the fasting and post-fed state $[20,21]$. Lower glucagon levels result in a reduction in post-hepatic glucose production. Additionally, the drug slows gastric emptying and improves satiety, thereby favouring weight loss $[20,21]$. Furthermore, semaglutide also 
demonstrates a beneficial effect on plasma lipids, lowering systolic blood pressure and reducing inflammation $[22,23]$.

Co-formulation of semaglutide with the absorption enhancer sodium N-(8-[2-hydroxybenzoyl] amino) caprylate (SNAC) increases gastric $\mathrm{pH}$ at the site of adherence on the gastric mucosa, allowing the peptide to safely exit the pill and be absorbed into the capillaries of the gastric mucosa [24]. This formulation offers people with T2D the option of an oral GLP-1 RA treatment with similar efficacy in reducing HbA1c and body weight, and with similar tolerability, as most injectable GLP-1 RAs [25].

The PIONEER (Peptide Innovation for Early Diabetes Treatment) trials, a global trial programme that enrolled approximately 9500 people with T2D on various background therapies across eight global clinical trials and two centres in Japan, demonstrated that oral semaglutide reduced HbA1c more than placebo, empagliflozin (a sodium-glucose transport protein 2 [SGLT2] inhibitor) or sitagliptin (a DPP-4 inhibitor), and showed similar HbA1c reductions to liraglutide. It also demonstrated greater reductions in body weight than placebo, sitagliptin or liraglutide, and a similar reduction to empagliflozin (Table 1) [13-15, 23, 26-30]. Furthermore, oral semaglutide had a safety profile consistent with that of subcutaneously injected GLP-1 RAs, with the most common adverse reactions being nausea, diarrhoea and, when used with insulin and/or sulfonylurea (SU), hypoglycaemia [31]. Additionally, a recent network meta-analysis demonstrated that orally administrated semaglutide had an efficacy similar to or better than semaglutide adminstered by subcutaneous injection, as well as similar tolerability [25].

With regards to $\mathrm{CV}$ outcomes, there are no completed CV outcomes trials (CVOTs) for oral semaglutide at present. The event-driven, double-blind PIONEER 6 trial, for which data analysis was halted as soon as it accumulated the necessary number of events to demonstrate noninferiority, was powered to determine excess CV risk associated with oral semaglutide and demonstrated noninferiority of oral semaglutide to placebo, ruling out an excess $\mathrm{CV}$ risk [27]. Post hoc analysis of PIONEER 6 and
SUSTAIN 6 showed that semaglutide consistently improved major adverse cardiovascular event (MACE) outcomes versus placebo, irrespective of the mode of administration [22]. In addition, a post hoc analysis of the LEADER, SUSTAIN 6 and PIONEER 6 trials demonstrated that semaglutide was particularly effective in reducing stroke incidence in patients with $\mathrm{T} 2 \mathrm{D}$ and high CV risk [32], a complication that is considered by people with T2D to be a particular concern [33]. PIONEER 6 was not powered to prove superiority and, thus, CV benefit. However, the ongoing SOUL CVOT (A Heart Disease Study of Semaglutide in Patients with Type 2 Diabetes; NCT03914326) will further inform on CV outcomes with oral semaglutide over a longer period of time and assess a larger population than PIONEER 6 [34]. The primary endpoint is time to the first occurrence of MACE, and this trial is powered for an assessment of superiority compared to placebo.

The findings highlighted in the clinical trial data indicate that oral semaglutide presents a valuable option for managing $\mathrm{T} 2 \mathrm{D}$, with the potential to expand the number of people with T2D benefiting from GLP-1 RAs. As such, oral semaglutide should be considered alongside other recommended OADs as second-line treatment in place of injectable GLP-1 RAs. While both GLP-1 RAs and SGLT2 inhibitors have demonstrated efficacy as a second-line treatment for people with T2D with heart failure (HF) and/or CKD comorbidities [35], SGLT2 inhibitors should be the preferred option for this group of people with T2D, since they provide a greater benefit, but at a significantly lower cost [36]. As a consensus group, we recommend that oral semaglutide should be considered for metabolic control over alternative options, such as DPP-4 inhibitors, SUs and thiazolidinediones (TZDs). Furthermore, in patients with T2D and HF/CKD, for whom SGLT2 inhibitors may be considered a preferential therapy, when additional glucose-lowering effects are required, such as in patients with estimated glomerular filtration rate (eGFR) $<60 \mathrm{ml} / \mathrm{min} / 1.73 \mathrm{~m}^{2}$, oral semaglutide should be considered as the preferred add-on OAD therapy. 


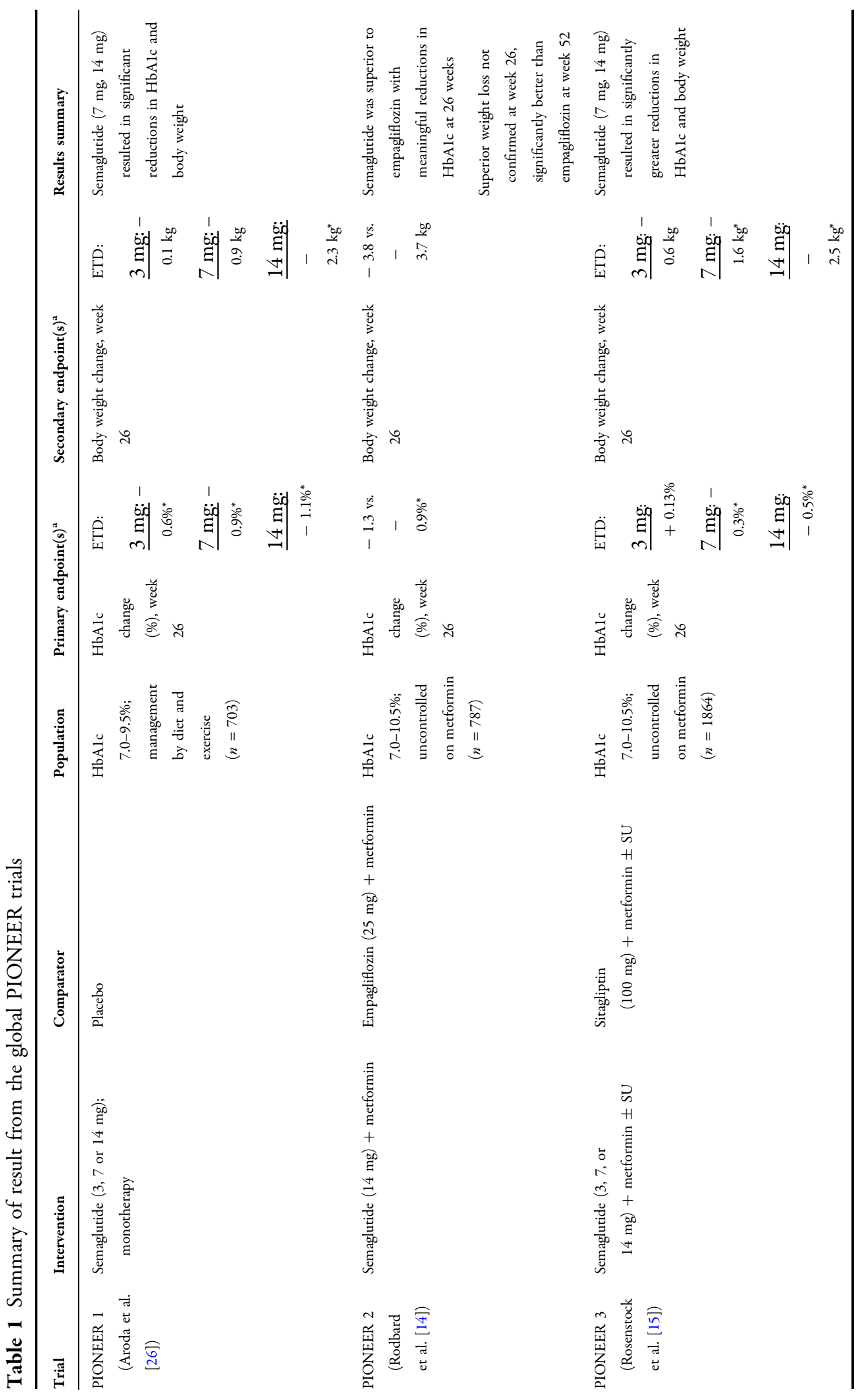




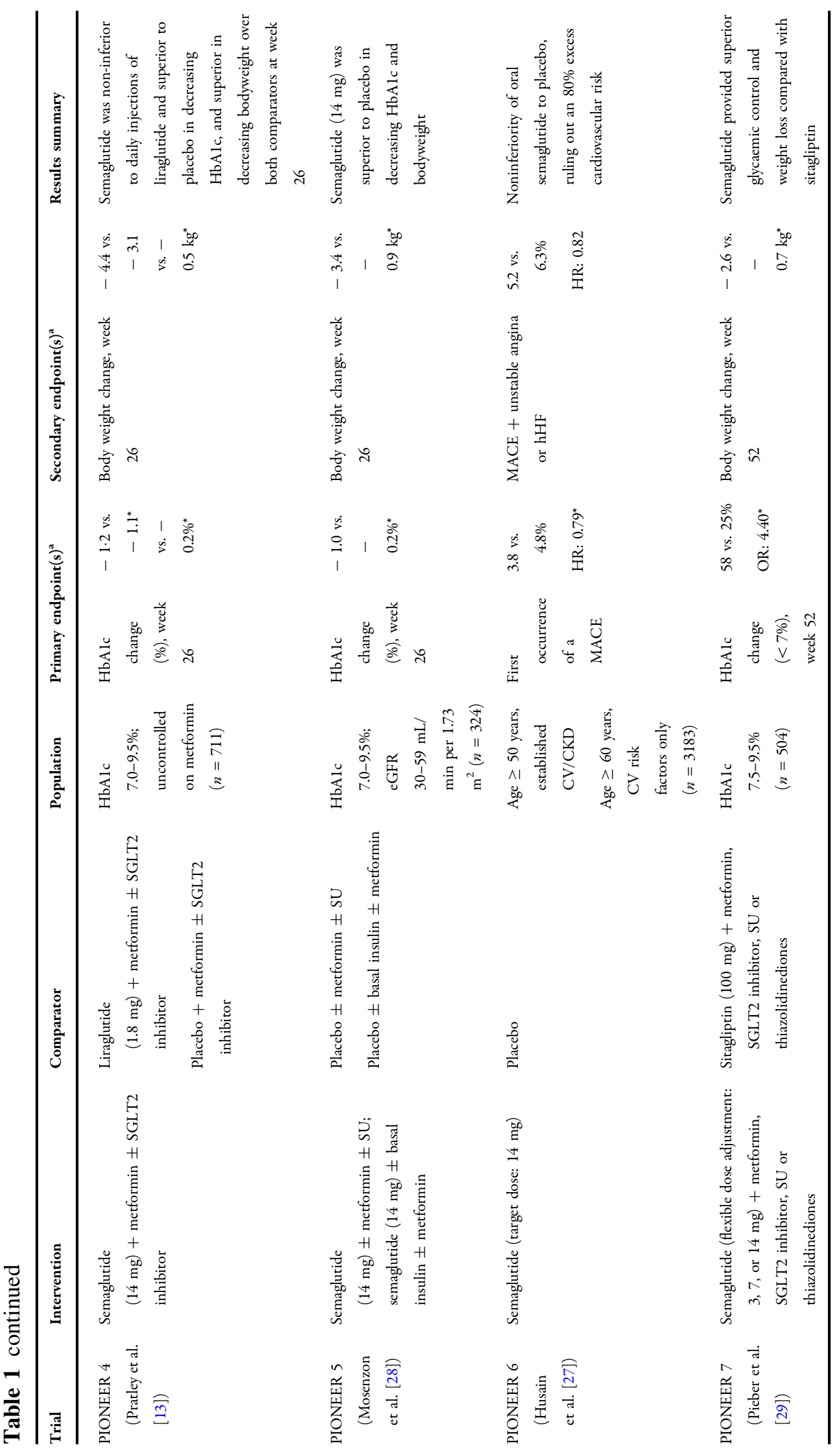




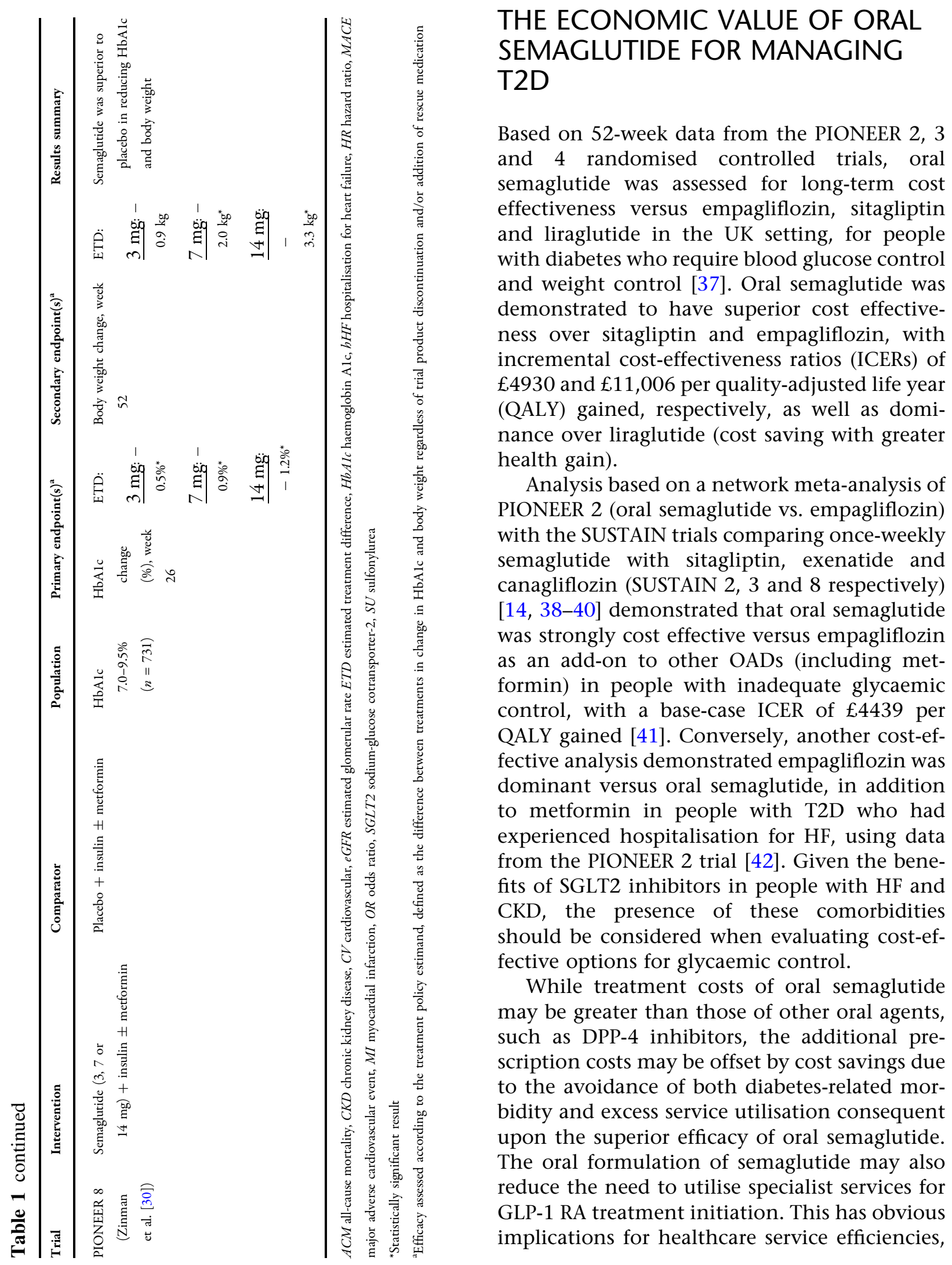


cost savings and capacity issues, whilst also addressing the burden of therapy inertia in people with T2D which has been further exacerbated during the COVID-19 pandemic.

Based on the above discussion, oral semaglutide can be considered as representing good value for money. There is, however, the wider value of oral semaglutide to the healthcare system, and associated stakeholders, beyond price and cost-effectiveness analyses to consider.

\section{THE VALUE OF ORAL SEMAGLUTIDE TO HEALTHCARE BEYOND PRICE}

While GLP-1RAs are among the most effective drugs for treating people with $\mathrm{T} 2 \mathrm{D}$, the reluctance of some patients to accept subcutaneous injections and the use of healthcare resources associated with injectables has resulted in low uptake of GLP-1RAs in clinical practice. Oral semaglutide may circumvent these issues and provide a new avenue for clinicians to enrol people with T2D on effective new therapies.

Being able to offer people with T2D the option of an oral GLP-1 RA can make it easier and quicker for people with T2D to be initiated on a GLP-1 RA, without the burden of considering an injectable route of administration. This has been of particular importance during the COVID-19 pandemic, as healthcare providers now have the option of an oral treatment without having to consider the logistical issues associated with initiating a person with T2D on an injectable therapy, whether by providing education virtually or by a physical visit for that purpose. As the simpler method of treatment administration can be explained more easily through virtual communications to people with diabetes compared with injection technique instruction, oral semaglutide facilitates the reduction of unnecessary attendance at clinics, reducing the risk of COVID-19 transmission from face-to-face appointments, including the visits of district nurse for those who are unable to self-inject.

Patient preference should also be taken into account when considering oral daily treatments versus weekly subcutaneous injections. Many people may struggle with the concept of using needles independently, and some may refuse to escalate treatment to injectables despite consultations explaining the benefits of GLP-1 RA over insulin, such as those with a severe phobia of needles. Furthermore, dexterity issues and hand osteoarthritis may also impede implementation of an injection technique, particularly in attaching a needle and sustaining the thumb pressure required to inject. Oral treatment in these instances may be more likely to be accepted and reduce the inertia surrounding the uptake of GLP-1 RAs.

Oral semaglutide may circumvent all of the issues described above and help to deliver value in the context of diabetes care not only to the healthcare system, but also to patients and their families.

\section{APPROPRIATE PRESCRIBING OF ORAL SEMAGLUTIDE IN T2D}

The selection of appropriate people with T2D for oral semaglutide treatment is critical for maximising both the risk-benefit profile and value associated with this treatment. Based on the PIONEER clinical trial data, as well as the label indication, and our expert consensus opinion, we have developed a checklist that serves as a tool to advise on the appropriate prescribing of oral semaglutide in people with T2D (Table 2). The checklist follows a traffic light system which recommends that people in the green section should be considered for treatment with oral semaglutide. This group includes adults (aged 18-74 years of age) with T2D without HF/CKD who require second-line treatment after metformin to control glycaemia. Oral semaglutide could also be considered in those individuals who do not have HF/ CKD but do have a history of stroke or transient ischemic attack (TIA), or have ASCVD or are at high $\mathrm{CV}$ risk.

In the PIONEER trials, there were no overall differences in the safety or efficacy of oral semaglutide between older adults aged $>75$ years with T2D and younger people with T2D [28]. However, the overall therapeutic experience of oral semaglutide in older adults is 
Table 2 Appropriate prescribing of oral semaglutide in patients with type 2 diabetes

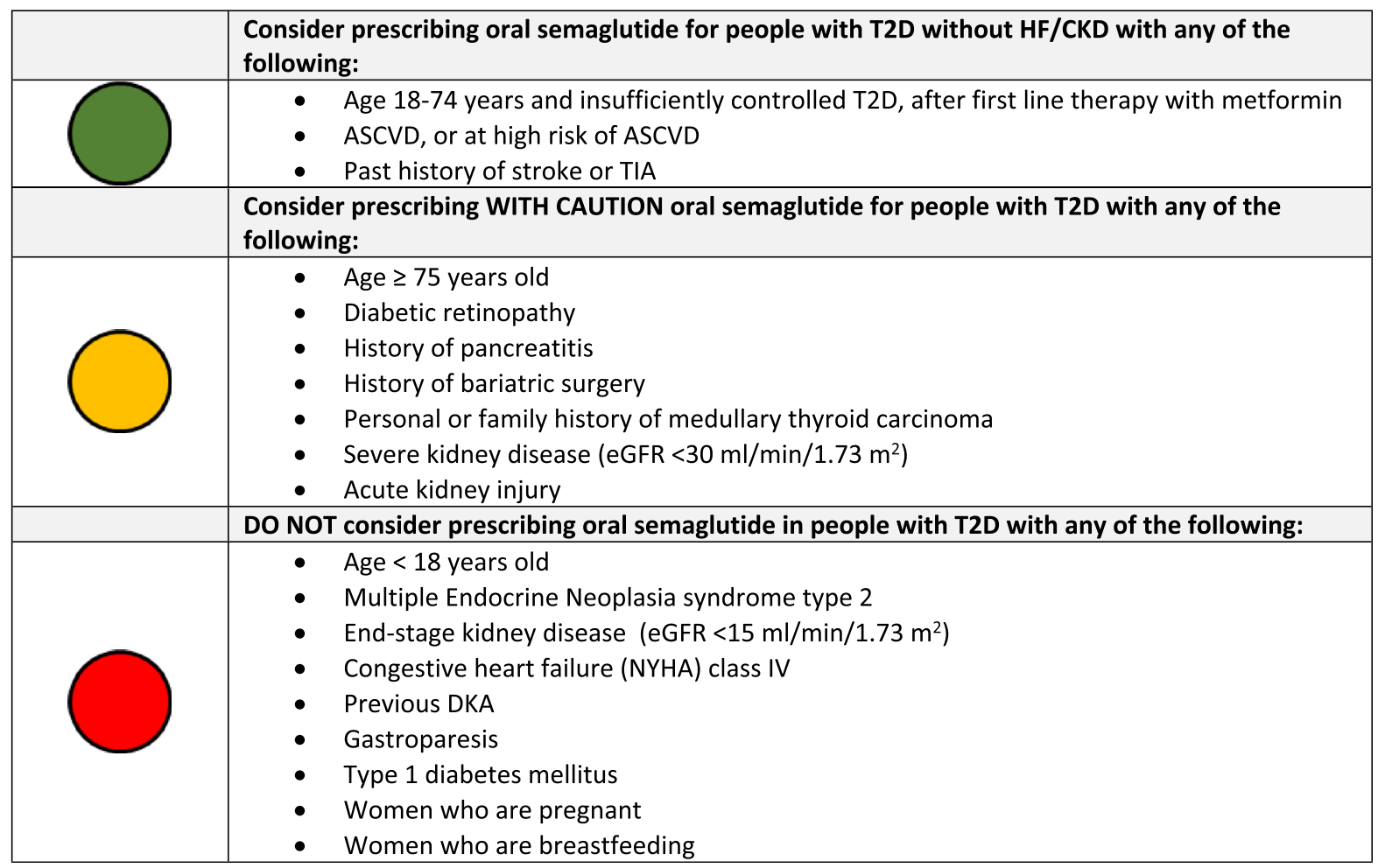

ASCVD atherosclerotic cardiovascular disease, CVD cardiovascular disease, DKA diabetic ketoacidosis, eGFR estimated glomerular filtration rate, NYHA New York Heart Association, TIA transient ischemic attack, T2D type 2 diabetes

relatively limited, and, as such, prescribing in this population should be considered with caution (amber section of the checklist).

The SUSTAIN 6 CVOT reported an increased incidence of diabetic retinopathy complications in patients randomised to semaglutide compared to placebo [43]. The majority of these adverse outcomes were attributed to a large and rapid fall in HbA1c during the first 16 weeks of the trial in individuals with pre-existing retinopathy who had a high $\mathrm{HbA1c}$ at baseline and were already receiving insulin [44]. This finding is supported by data from the LEADER and REWIND trials that demonstrated an increase in retinopathy events associated with a large, sudden fall in $\mathrm{HbA} 1 \mathrm{c}$ in participants who were randomised to liraglutide and dulaglutide, respectively $[45,46]$. Therefore, in alignment with other published guidance [47], we recommended that oral semaglutide should be prescribed with caution in people with T2D with a history of diabetic retinopathy requiring active ophthalmology follow-up, receiving insulin treatment and with poor glycaemic control (HbA1c > $91 \mathrm{mmol} / \mathrm{mol}$ [0.5\%]), and monitored closely, particularly if there has been no recent retinal screening.

Treatment with oral semaglutide is not recommended in people with end-stage kidney disease (eGFR $<15 \mathrm{ml} / \mathrm{min} / 1.73 \mathrm{~m}^{2}$ ); however, pharmacokinetic-pharmacodynamic studies have demonstrated no significant effect of declining eGFR on the pharmacological profile [48]. The PIONEER clinical trial programme largely excluded patients with eGFR $<30 \mathrm{ml} /$ $\min / 1.73 \mathrm{~m}^{2}$; hence experience in this group is limited. While semaglutide may be prescribed in patients with eGFR of $15-30 \mathrm{ml} / \mathrm{min} / 1.73 \mathrm{~m}^{2}$, due to limited experience in this group we would suggest proceeding with caution in such 
patients. Similarly, in patients with acute kidney injury, the use of oral semaglutide should be used with caution, largely from the perspective that should significant gastrointestinal effects occur, these could potentiate dehydration and exacerbate kidney injury.

While there is no definitive evidence of a causal association between semaglutide and acute pancreatitis, we would advocate the use of oral semaglutide with caution in patients with prior history of pancreatitis, be that idiopathic or precipitated by a specific aetiological factor, such as, for example, gallstone pancreatitis.

Patients with T2D and with prior bariatric surgery may be considered as appealing candidates for a GLP-1 RA. A study assessing the safety and efficacy of liraglutide in people with persistent or recurrent T2D after bariatric surgery demonstrated that liraglutide treatment was associated with a difference of $-1.22 \%$ (95\% confidence interval -19.7 to -7.0 ; $p=0.0001)$ in HbA1c change from baseline to 26 weeks and no difference in the frequency of adverse events, compared with placebo [49]. The effects of bariatric surgery and in particular the different surgical modalities on the absorption, and thus clinical efficacy, of oral semaglutide have not been studied, as such we would recommend the use of oral semaglutide with caution in patients with $\mathrm{T} 2 \mathrm{D}$ and prior bariatric surgery.

Oral semaglutide increases the incidence of thyroid C-cell tumours in certain settings. A meta-analysis of once-weekly GLP-1 RAs in people with T2D showed no increased risk in comparison with other treatments [50]; however, there are documented cases of medullary thyroid carcinoma in people with T2D treated with the GLP-1 RA liraglutide, although a causal relationship is not established. We therefore recommend caution in the use of oral semaglutide in patients with a personal or family history of medullary thyroid carcinoma. However, we do not recommend prescribing oral semaglutide in people with T2D with multiple endocrine neoplasia syndrome type 2 , guidance which is in accordance with the licence.

Oral semaglutide is not indicated as a treatment in people with type 1 diabetes, for the treatment of diabetic ketoacidosis (DKA), or in those with a history of DKA [51]. DKA has been documented in insulin-dependent people with T2D that initiate GLP-1 RAs, who rapidly discontinue insulin therapy ahead of initiation.

The safety and efficacy of oral semaglutide has also not been established in the paediatric setting and is therefore not indicated for those aged $<18$ years. There is also no documented experience of treating people with congestive HF, NHYA (New York Heart Association) class VI, and as such treatment in this population should not be considered.

Oral semaglutide is contraindicated in pregnant women due to the potential risk to the foetus; treatment should only be considered in instances where treatment benefit justifies the risk to the foetus. Oral semaglutide should be discontinued at least 2 months before planned pregnancy due to the long washout period associated with this drug [52]. Improved glycaemic control and weight loss afforded by oral semaglutide has the potential to improve fertility, and patients should be informed of this benefit if they are planning a pregnancy in the future, although they should be warned to avoid conception whilst taking the drug. Oral semaglutide is not recommended in women who are lactating. There are no data regarding the presence of semaglutide in breast milk, although in animal studies it was demonstrated that semaglutide and its excipients or associated metabolites were present.

GLP-1 RAs have a well-recognised effect on gastric emptying. Consequently, we would not advocate the use of oral semaglutide in people with T2D and established gastroparesis.

\section{PRACTICAL CONSIDERATIONS FOR OPTIMAL USE OF ORAL SEMAGLUTIDE}

Due to the formulation of oral semaglutide, clinicians need to be aware of specific considerations in its administration. As absorption of oral semaglutide is reduced if taken with food and large volumes of fluid, with higher levels of absorption from longer post-dose fasting period $[24,52]$, individuals prescribed oral semaglutide 
should be informed that once-daily dosing is required at least $30 \mathrm{~min}$ before eating, drinking or taking other oral medicinal products. They should additionally be advised that the tablet should be swallowed whole (and should not be split, crushed or chewed) with up to half a glass of tap water (approximately $120 \mathrm{~mL} / 4$ fluid oz) [52] to ensure it traverses the oesophagus (avoiding irritancy) and reaches the stomach to be absorbed. It is also possible that consuming more than the recommended $120 \mathrm{~mL}$ water may also adversely impact absorption [24]. Thus, to ensure adherence to this instruction, it is imperative that the pack label includes the volume of water in the administration instructions. Furthermore, given the importance of adhering to the administration directions in achieving an effective bioavailability, it is vital that healthcare professionals, including pharmacists, effectively communicate these instructions and their importance to those initiating treatment with oral semaglutide.

Gradual dose escalation is recommended (and if a dose is missed, the missed dose should be skipped and the next dose should be taken the following day), with $3 \mathrm{mg}$ tablets taken daily in the first month, increasing to a maintenance daily dose of $7 \mathrm{mg}$ tablets in the second month and thereafter. After at least 1 month of $7 \mathrm{mg}$ tablets once daily, the dose can be increased to a maintenance daily dose of $14 \mathrm{mg}$ tablets to further improve glycaemic control if required (taking two $7 \mathrm{mg}$ tablets to achieve the effect of one $14 \mathrm{mg}$ dose has not been studied, but would increase the cost, and so is not recommended). This escalation will minimise gastrointestinal adverse reactions during the initial stages of treatment [21]. While these reactions are typically mild to moderate and abate over time [20, 21], monitoring of renal function in people with T2D experiencing severe gastrointestinal reactions is recommended in case of potential dehydration.

Treatment response should be assessed initially at 3 months to identify those with weaker response than expected, although full response will not be observed until 6 months after first dose. Absorption may vary between individuals, and in those achieving insufficient response adherence should be examined and treatment discontinuation considered. No dose adjustments are necessary in those with severe renal impairment $\left(e G F R<15 \mathrm{ml} / \mathrm{min} / 1.73 \quad \mathrm{~m}^{2}\right)$, upper gastrointestinal disease or hepatic impairment, as the presence of these comorbidities does not affect the pharmacokinetics of oral semaglutide [28].

Medication management strategies to mitigate potential adverse events and to support treatment continuation are important and could include the incorporation of treatment pauses or dose reduction when tolerability issues arise, alongside patient education [53]. The most frequent side-effects from using GLP-1 RAs are gastrointestinal (GI). Individuals need to be warned of the possibility of nausea and vomiting but should also be reassured that these are usually mild to moderate in intensity and dissipate with time. The importance of continuing with treatment should be reiterated, and slowed titration may be considered in severe cases until GI side effects have resolved. Individuals should also be provided with education regarding smaller meal sizes and reduced carbohydrate and fat content in their diets, as well as to generally reinforce healthy eating to achieve best outcomes with this drug, thereby reducing the incidence of nausea, vomiting and other GI side effects.

Oral semaglutide alone is not associated with an increased risk of hypoglycaemia. However, when it is used in combination with agents that may cause hypoglycaemia, such as, for example, SUs or exogenous insulin, the potential improvement in glucose control may be associated with hypoglycaemia, and patients should be educated accordingly. In order to reduce potential hypoglycaemia risk, our consensus viewpoint is that in patients with $\mathrm{Hb} 1 \mathrm{Ac}<$ $7.5 \%$, a dose reduction of insulin and SU insulin of up to $50 \%$ may be considered, while in those with $\mathrm{HbA} 1 \mathrm{c}<8 \%$, up to $20 \%$ dose reduction may be considered.

Further consideration should be given when prescribing oral semaglutide to people already taking oral medications that require ingestion on an empty stomach, such as bisphosphonates and levothyroxine. Oral bisphosphonates should not be taken with oral semaglutide, and instead annual intravenous infusions should be 
considered as an alternative. As administration of oral semaglutide in people taking thyroxine can result in excess thyroxine absorption [54], monitoring of thyroid parameters should be undertaken when treating people with T2D with semaglutide if they are also prescribed levothyroxine. In addition, dosing schedules should be altered, such as administrating levothyroxine at night, in order to minimise any potential impact on thyroid hormone levels that may occur if oral semaglutide and levothyroxine are administered at the same time of day. It is important that these issues are discussed with the individual to ensure that appropriate dosing of both medications is compatible with lifestyle, thus optimising the potential for therapy adherence. The REVISE study demonstrated the importance of educating people with diabetes on the relative merits of the available options to ensure that they are able to initiate new therapies that fit their lifestyle [55].

\section{SUMMARY}

In the PIONEER clinical development programme, oral semaglutide demonstrated significant $\mathrm{HbA1c}$ and weight reductions, and as such may be considered as the most efficacious oral therapy with respect to metabolic control in people with T2D. The evolution of GLP-1 RA therapy into an oral formulation will enable many more people with T2D to access the therapeutic benefits of this treatment class and help address the well-described issue of therapy inertia in this population. The COVID-19 pandemic has only served to further exacerbate the problems of therapy inertia in the management of people with T2D, and the availability of oral semaglutide at this time further enhances the clinical and economic value of this treatment in the management of people with T2D.

\section{ACKNOWLEDGEMENTS}

Funding. This manuscript was supported by a grant from Novo Nordisk in terms of medical writing and Rapid Service Fee. Novo Nordisk has not influenced the content of the publication or been involved in the writing of this publication. Novo Nordisk has reviewed this document for factual accuracy only and the views expressed in this publication are those of the authors.

Authorship. All named authors meet the International Committee of Medical Journal Editors (ICMJE) criteria for authorship for this article, take responsibility for the integrity of the work as a whole, and have given their approval for this version to be published.

Author Contributions. All authors have been involved in study concept and design and have contributed to discussions around manuscript content. The first draft of the manuscript was written by Angharad R Morgan and Marc Evans. All authors commented on this draft and contributed to subsequent versions of the manuscript. All authors read and approved the final manuscript for publication.

Medical Writing, Editorial, and Other Assistance. We thank Peter Gabb of Health Economics Outcomes Research Ltd. for providing medical writing and editorial support. Support for this assistance was funded by Novo Nordisk UK.

Disclosures. Marc Evans reports honoraria from AstraZeneca, Novo Nordisk, Takeda and Napp, and research support from Novo Nordisk outside the submitted work. Angharad R Morgan is an employee of Health Economics and Outcomes Research Ltd., Cardiff, UK. Health Economics and Outcomes Research Ltd. received fees from Novo Nordisk in relation to this study. Stephen C Bain reports receiving lecture and advisory board fees from AstraZeneca, Boehringer Ingelheim, Eli Lilly, Merck Sharp and Dohme, Novo Nordisk, Roche, Sanofi-Aventis; has shares in Glycosmedia, which carries sponsorship declared on site; and provides expert advice to the All-Wales Medicines Strategy Group and the National Institute for Health and Care Excellence (UK). Sarah Davies has received honorarium for speaking or grants for attending meetings from 
AstraZeneca, Boehringer Ingelheim, Lilly, Merck Sharp and Dohme, Napp, Novo Nordisk and Takeda. Debbie Hicks has no conflicts of interest to declare. Pam Brown received funding for advisory boards, ad hoc consultancy, conference registration and travel and delivery of medical education from Abbott, Astra Zeneca, Boehringer Ingelheim, Eli Lilly, Janssen, MSD, Napp and Novo Nordisk. Zaheer Yousef has received lecture fees and honoraria from Boehringer Ingelheim, Lilly, Astra Zeneca, Novartis and Pfizer. Umesh Dashora has no conflicts of interest to declare. Adie Viljoen has conducted research studies, served as advisor for and/or received lecture honoraria from Amgen, AstraZeneca, Boehringer, Daiichi, Eli Lilly, Napp, Novartis, Novo Nordisk, MannKind, Pfizer, Regeneron, Sanofi, Takeda and Tosoh. Hannah Beba has received speaker honoraria and consultancy fees from Astra Zeneca, Boehringer Ingelheim, Eli Lilly, Napp, Novo Nordisk, Sanofi and Takeda. W David Strain has received speaker honoraria and unrestricted educational grants from Astra Zeneca, Bayer, Boehringer Ingelheim, Eli Lilly, Glaxo SmithKline, Merck, Napp, Novartis, Novo Nordisk and Takeda. W David Strain is supported by the NIHR Exeter Clinical Research Facility and the South West Peninsula NIHR Applied Research Collaboration (PenARC). The views expressed in this publication are those of the author(s) and not necessarily those of the NIHR Exeter Clinical Research Facility, the NHS, the NIHR or the Department of Health in England.

Compliance with Ethics Guidelines. This article is based on previously published studies and does not contain any new studies with human participants or animals performed by any of the authors.

Data Availability. Data sharing is not applicable to this article as no datasets were generated or analysed during the current study.

Open Access. This article is licensed under a Creative Commons Attribution-NonCommercial 4.0 International License, which permits any non-commercial use, sharing, adaptation, distribution and reproduction in any medium or format, as long as you give appropriate credit to the original author(s) and the source, provide a link to the Creative Commons licence, and indicate if changes were made. The images or other third party material in this article are included in the article's Creative Commons licence, unless indicated otherwise in a credit line to the material. If material is not included in the article's Creative Commons licence and your intended use is not permitted by statutory regulation or exceeds the permitted use, you will need to obtain permission directly from the copyright holder. To view a copy of this licence, visit http:// creativecommons.org/licenses/by-nc/4.0/.

\section{REFERENCES}

1. International Diabetes Federation (IDF). IDF diabetes atlas. Ninth edition 2019. 2019. https://www. diabetesatlas.org/upload/resources/material/ 20200302_133351_IDFATLAS9e-final-web.pdf. Accessed 28 July 2021.

2. Sarwar N, Gao P, Seshasai SR, et al. Diabetes mellitus, fasting blood glucose concentration, and risk of vascular disease: a collaborative meta-analysis of 102 prospective studies. Lancet. 2010;375(9733): 2215-22.

3. Paul SK, Klein K, Thorsted BL, Wolden ML, Khunti $\mathrm{K}$. Delay in treatment intensification increases the risks of cardiovascular events in patients with type 2 diabetes. Cardiovasc Diabetol. 2015;14:100.

4. NHS Digital. National Diabetes Audit (NDA) 2020-2021 quarterly report for England, Clinical Commissioning Groups and GP practices-PROVISIONAL. 2021. https://digital.nhs.uk/data-andinformation/publications/statistical/nationaldiabetes-audit/care-processes-and-treatmenttargets-3rd-quarter-january-december-2020-datarelease. Accessed 14 Sep 2021.

5. DeFronzo RA. Combination therapy with GLP-1 receptor agonist and SGLT2 inhibitor. Diabetes Obes Metab. 2017;19(10):1353-62.

6. DeFronzo RA, Ferrannini E, Groop L, et al. Type 2 diabetes mellitus. Nat Rev Dis Prim. 2015;1:15019.

7. Mardetko N, Nabergoj Makovec U, Locatelli I, Janez A, Kos M. Uptake of new antidiabetic medicines in 11 European countries. BMC Endocr Disord. 2021;21(1):127. 
8. Matza LS, Curtis SE, Jordan JB, Adetunji O, Martin SA, Boye KS. Physician perceptions of GLP-1 receptor agonists in the UK. Curr Med Res Opin. 2016;32(5):857-64.

9. Royal College of General Practitioners (RCGP). RCGP Guidance on workload prioritisation during COVID-19. 2020. https://www.rcgp.org.uk/-/ media/Files/Policy/A-Z-policy/2020/covid19/ RCGP\%20guidance/202003233RCGPGuidance prioritisationroutineworkduringCovidFINAL. Accessed 14 Sep 2021.

10. Quinn LM, Davies MJ, Hadjiconstantinou M. Virtual consultations and the role of technology during the COVID-19 pandemic for people with type 2 diabetes: the UK perspective. J Med Internet Res. 2020;22(8):e21609.

11. Diabetes UK. Diabetes care during the COVID-19 pandemic; position statement. December 2020. https://diabetes-resources-production.s3.eu-west-1. amazonaws.com/resources-s3/public/2020-12/ Position\%20statement\%20on\%20diabetes\% 20care\%20during\%20the\%20Covid-19\% 20pandemic.pdf. Accessed 28 Jul 2021.

12. Aloudah NM, Scott NW, Aljadhey HS, Araujo-Soares V, Alrubeaan KA, Watson MC. Medication adherence among patients with type 2 diabetes: a mixed methods study. PLoS ONE. 2018;13(12):e0207583.

13. Pratley R, Amod A, Hoff ST, et al. Oral semaglutide versus subcutaneous liraglutide and placebo in type 2 diabetes (PIONEER 4): a randomised, doubleblind, phase 3a trial. Lancet. 2019;394(10192): 39-50.

14. Rodbard HW, Rosenstock J, Canani LH, et al. Oral semaglutide versus empagliflozin in patients with type 2 diabetes uncontrolled on metformin: the PIONEER 2 trial. Diabetes Care. 2019;42(12): 2272-81.

15. Rosenstock J, Allison D, Birkenfeld AL, et al. Effect of additional oral semaglutide vs sitagliptin on glycated hemoglobin in adults with type 2 diabetes uncontrolled with metformin alone or with sulfonylurea: the PIONEER 3 randomized clinical trial. JAMA. 2019;321(15):1466-80.

16. National Institute for Health and Care Excellence (NICE). NICE guideline [NG28] Type 2 diabetes in adults: management. 2015. Updated 2021. https:// www.nice.org.uk/guidance/ng28. Accessed 10 Aug 2021.

17. Dashora U, Wheatcroft SB, Winocour P, et al. How should we manage people with diabetes in a holistic manner in 2021. Consensus guidance from Cardiology Renal and Metabolic (CaReMe-UK) group. 2021. https://www.guidelinesinpractice.co.uk/ diabetes/manage-diabetes-and-comorbidities-witha-joined-up-strategy/456004.article. Accessed date 23 Aug 2021.

18. Cosentino F, Grant PJ, Aboyans V, et al. 2019 ESC Guidelines on diabetes, pre-diabetes, and cardiovascular diseases developed in collaboration with the EASD. Eur Heart J. 2020;41(2):255-323.

19. Buse JB, Wexler DJ, Tsapas A, et al. 2019 Update to: management of hyperglycemia in type 2 diabetes, 2018. A consensus report by the American Diabetes Association (ADA) and the European Association for the Study of Diabetes (EASD. Diabetes Care. 2020;43(2):487-93.

20. Lavernia F, Blonde L. Clinical review of the efficacy and safety of oral semaglutide in patients with type 2 diabetes compared with other oral antihyperglycemic agents and placebo. Postgrad Med. 2020;132(Suppl 2):15-25.

21. Wright EE Jr, Aroda VR. Clinical review of the efficacy and safety of oral semaglutide in patients with type 2 diabetes considered for injectable GLP-1 receptor agonist therapy or currently on insulin therapy. Postgrad Med. 2020;132(Suppl 2):26-36.

22. Husain M, Bain SC, Jeppesen OK, et al. Semaglutide (SUSTAIN and PIONEER) reduces cardiovascular events in type 2 diabetes across varying cardiovascular risk. Diabetes Obes Metab. 2020;22(3):442-51.

23. Thethi TK, Pratley R, Meier JJ. Efficacy, safety and cardiovascular outcomes of once-daily oral semaglutide in patients with type 2 diabetes: the PIONEER programme. Diabetes Obes Metab. 2020;22(8):1263-77.

24. Buckley ST, Bækdal TA, Vegge A, et al. Transcellular stomach absorption of a derivatized glucagon-like peptide-1 receptor agonist. Sci Transl Med. 2018;10(467):eaar7047.

25. Chubb B, Gupta P, Gupta J, Nuhoho S, Kallenbach $\mathrm{K}$, Orme M. Once-daily oral semaglutide versus injectable GLP-1 RAs in people with type 2 diabetes inadequately controlled on basal insulin: systematic review and network meta-analysis. Diabetes Ther. 2021;12(5):1325-39.

26. Aroda VR, Rosenstock J, Terauchi Y, Altuntas Y, Lalic NM, Morales Villegas EC, et al. PIONEER 1: randomized clinical trial of the efficacy and safety of oral semaglutide monotherapy in comparison with placebo in patients with type 2 diabetes. Diabetes Care. 2019;42(9):1724-32.

27. Husain M, Birkenfeld AL, Donsmark M, et al. Oral semaglutide and cardiovascular outcomes in patients with type 2 diabetes. $\mathrm{N}$ Engl J Med. 2019;381(9):841-51. 
28. Mosenzon O, Blicher TM, Rosenlund S, et al. Efficacy and safety of oral semaglutide in patients with type 2 diabetes and moderate renal impairment (PIONEER 5): a placebo-controlled, randomised, phase 3a trial. Lancet Diabetes Endocrinol. $2019 ; 7(7): 515-27$.

29. Pieber TR, Bode B, Mertens A, et al. Efficacy and safety of oral semaglutide with flexible dose adjustment versus sitagliptin in type 2 diabetes (PIONEER 7): a multicentre, open-label, randomised, phase $3 \mathrm{a}$ trial. Lancet Diabetes Endocrinol. 2019;7(7):528-39.

30. Zinman B, Aroda VR, Buse JB, et al. Efficacy, safety, and tolerability of oral semaglutide versus placebo added to insulin with or without metformin in patients with type 2 diabetes: the PIONEER 8 trial. Diabetes Care. 2019;42(12):2262-71.

31. Rodbard HW, Dougherty T, Taddei-Allen P. Efficacy of oral semaglutide: overview of the PIONEER clinical trial program and implications for managed care. Am J Manag Care. 2020;26:S335-43.

32. Strain WD, Holst AGSR, Saevereid HA, James MA. Effects of liraglutide and semaglutide on stroke subtypes in patients with type 2 diabetes: a post hoc analysis of the LEADER, SUSTAIN 6 and PIONEER 6 trials. 2020. https://www.easd.org/virtualmeeting/ home.html\#!resources/effects-of-liraglutide-andsemaglutide-on-stroke-subtypes-in-patients-withtype-2-diabetes-a-post-hoc-analysis-of-the-leadersustain-6-and-pioneer-6-trials. Accessed date 22 Sep 2021.

33. Diabetes UK. Survey reveals patients' concerns about diabetes complications. 2009. https://www. diabetes.org.uk/about_us/news_landing_page/ survey-reveals-patients-concerns-about-diabetescomplications\#: :text=The $\% 20$ results $\% 20$ of $\%$ 20a\%20Diabetes, have\%20experienced $\% 20$ sexual $\%$ 20dysfunction\%3B\%20just. Accessed 20 Aug 2021.

34. ClinicalTrials.gov. NCT03914326. A heart disease study of semaglutide in patients with type 2 diabetes (SOUL). 2021. https://clinicaltrials.gov/ct2/ show/NCT03914326

35. Palmer SC, Tendal B, Mustafa RA, et al. Sodiumglucose cotransporter protein-2 (SGLT-2) inhibitors and glucagon-like peptide-1 (GLP-1) receptor agonists for type 2 diabetes: systematic review and network meta-analysis of randomised controlled trials. BMJ. 2021;372:m4573.

36. Wysham $\mathrm{CH}$, Pilon $\mathrm{D}$, Ingham $\mathrm{M}$, et al. Hba1C control and cost-effectiveness in patients with type 2 diabetes mellitus initiated on canagliflozin or a glucagon-like peptide 1 receptor agonist in a realworld setting. Endocr Pract. 2018;24(3):273-88.
37. Bain SC, Hansen BB, Malkin SJP, et al. Oral semaglutide versus empagliflozin, sitagliptin and liraglutide in the UK: long-term cost-effectiveness analyses based on the PIONEER clinical trial programme. Diabetes Ther. 2020;11(1):259-77.

38. Ahmann AJ, Capehorn M, Charpentier G, et al. Efficacy and safety of once-weekly semaglutide versus exenatide er in subjects with type 2 diabetes (SUSTAIN 3): a 56-week, open-label. Randomized Clin Trial Diabetes Care. 2018;41(2):258-66.

39. Ahrén B, Masmiquel L, Kumar H, et al. Efficacy and safety of once-weekly semaglutide versus once-daily sitagliptin as an add-on to metformin, thiazolidinediones, or both, in patients with type 2 diabetes (SUSTAIN 2): a 56-week, double-blind, phase 3a, randomised trial. Lancet Diabetes Endocrinol. 2017;5(5):341-54.

40. Lingvay I, Catarig A-M, Frias JP, et al. Efficacy and safety of once-weekly semaglutide versus daily canagliflozin as add-on to metformin in patients with type 2 diabetes (SUSTAIN 8): a double-blind, phase $3 \mathrm{~b}$, randomised controlled trial. Lancet Diabetes Endocrinol. 2019;7(11):834-44.

41. Capehorn M, Hallén N, Baker-Knight J, Glah D, Hunt B. Evaluating the cost-effectiveness of onceweekly semaglutide $1 \mathrm{mg}$ versus empagliflozin 25 $\mathrm{mg}$ for treatment of patients with type 2 diabetes in the UK setting. Diabetes Ther. 2021;12(2):537-55.

42. Ramos M, Cummings MH, Ustyugova A, Raza SI, de Silva SU, Lamotte M. Long-term cost-effectiveness analyses of empagliflozin versus oral semaglutide, in addition to metformin, for the treatment of type 2 diabetes in the UK. Diabetes Ther. 2020;11(9): 2041-55.

43. Marso SP, Bain SC, Consoli A, et al. Semaglutide and cardiovascular outcomes in patients with type 2 diabetes. N Engl J Med. 2016;375(19):1834-44.

44. Vilsbøll T, Bain SC, Leiter LA, et al. Semaglutide, reduction in glycated haemoglobin and the risk of diabetic retinopathy. Diabetes Obes Metab. 2018;20(4):889-97.

45. Gerstein HC, Colhoun HM, Dagenais GR, et al. Dulaglutide and cardiovascular outcomes in type 2 diabetes (REWIND): a double-blind, randomised placebo-controlled trial. Lancet. 2019;394(10193): 121-30.

46. Marso SP, Daniels GH, Brown-Frandsen $\mathrm{K}$, et al. Liraglutide and cardiovascular outcomes in type 2 diabetes. N Engl J Med. 2016;375(4):311-22.

47. Bain SC. GLP-1 receptor agonists and diabetic retinopathy. Diabetes Prim Care. 2021;23(4):290. 
48. Granhall C, Søndergaard FL, Thomsen M, Anderson TW. Pharmacokinetics, safety and tolerability of oral semaglutide in subjects with renal impairment. Clin Pharmacokinet. 2018;57(12):1571-80.

49. Miras AD, Pérez-Pevida B, Aldhwayan M, et al. Adjunctive liraglutide treatment in patients with persistent or recurrent type 2 diabetes after metabolic surgery (GRAVITAS): a randomised, doubleblind, placebo-controlled trial. Lancet Diabetes Endocrinol. 2019;7(7):549-59.

50. Bethel MA, Patel RA, Merrill P, et al. Cardiovascular outcomes with glucagon-like peptide-1 receptor agonists in patients with type 2 diabetes: a metaanalysis. Lancet Diabetes Endocrinol. 2018;6(2): 105-13.

51. Brunton SA, Mosenzon O, Wright EE Jr. Integrating oral semaglutide into clinical practice in primary care: for whom, when, and how? Postgrad Med. 2020;132(Suppl 2):48-60.
52. European Medicines Agency. Rybelsus. Summary of Product Characteristics. 2020. https://www.ema. europa.eu/en/documents/product-information/ rybelsus-epar-product-information_en.pdf. Accessed 23 Aug 2021.

53. Aroda VR, Bauer R, Davies AL, et al. 664-P: Incorporating treatment pauses, dosing flexibility, and education to support GLP-1RA therapy persistence. Diabetes. 2021;70(Suppl 1):664-P.

54. Hauge C, Breitschaft A, Hartoft-Nielsen M, Jensen S, Bækdal T. SAT-140 A drug-drug interaction trial of oral semaglutide with levothyroxine and multiple coadministered tablets. J Endocr Soc. 2019;3:SAT140 .

55. Boye K, Ross M, Mody R, Konig M, Gelhorn H. Patients' preferences for once-daily oral versus once-weekly injectable diabetes medications: the REVISE study. Diabetes Obes Metab. 2021;23(2): 508-19. 